\title{
Accretion powered spherical wind in general relativity
}

\author{
Tapas K. Das ${ }^{1,2}$ \\ 1 Astronomy Unit, Queen Mary \& Westfield College, Mile End Rd, London E1 4NS, UK \\ e-mail: T.Das@qmw.ac.uk \\ 2 Inter University Centre For Astronomy And Astrophysics, Post Bag 4 Ganeshkhind, Pune 411 007, India \\ e-mail: tapas@iucaa.ernet.in
}

Received 9 May 2001 / Accepted 20 June 2001

\begin{abstract}
Using full general relativistic calculations, we investigate the possibility of generation of mass outflow from spherical accretion onto non-rotating black holes. Introducing a relativistic hadronic-pressure-supported steady, standing, spherically-symmetric shock surface around a Schwarzschild black hole as the effective physical barrier that may be responsible for the generation of spherical wind, we calculate the mass outflow rate $R_{\mathrm{m}}$ in terms of three accretion parameters and one outflow parameter by simultaneously solving the set of general relativistic hydrodynamic equations describing spherically symmetric, transonic, polytropic accretion and wind around a Schwarzschild black hole. Not only do we provide a sufficiently plausible estimation of $R_{\mathrm{m}}$, we also successfully study the dependence and variation of this rate on various physical parameters governing the flow. Our calculation indicates that independent of initial boundary conditions, the baryonic matter content of this shock-generated wind always correlates with post-shock flow temperature.
\end{abstract}

Key words. accretion, accretion discs - outflow - black hole physics - general relativity - hydrodynamics

\section{Introduction}

Accretion onto massive celestial bodies is believed to be responsible for energy generation mechanism in many astrophysical objects including galactic binary systems and extra-galactic objects like Quasars and AGNs. Investigation of transonic, spherically symmetric accretion onto gravitating astrophysical objects was carried out from a purely Newtonian approach as well as using a pseudo-general relativistic and complete general relativistic framework (Hoyle \& Littleton 1939; Bondi 1952; Michel 1972; Shapiro 1973a,b; Blumenthal \& Mathews 1976; Begelman 1978; Brinkmann 1980; Malec 1999; Das 2001; Das \& Sarkar 2001; Sarkar \& Das 2001). However, in works mentioned above, spherical accretion was shown to exhibit essentially directed motion (radial inflow) which smoothly passes through a sonic point and no discontinuity in accretion onto a black hole was taken into account. Nevertheless, it was understood that accretion onto a black hole could be efficient in producing radiative energy if the directed in-fall motion could be randomized close to the hole by some suitable mechanism the best of which would be shock formation by some means. For radial accretion, a novel mechanism has been proposed (Protheros \& Kazanas 1983; Kazanas \& Ellision 1986,

Send offprint requests to: Tapas K. Das, e-mail: tapas@iucaa.ernet.in hereafter PK83 and KE86 respectively) in which the kinetic energy of spherically accreting material has been randomized by proposing a steady, collision-less, relativistic hadronic-pressure-supported spherically symmetric shock around a Schwarzschild black hole which produces a nonthermal spectrum of high energy (relativistic) protons. A thermal distribution of particles may also be produced at the shock which radiates by thermal bremstrahlung but only the radiation resulting from the non-thermal (which might actually contain most of the energy, see Axford 1981) was considered in their model. Since radial in-fall (freely falling situation) in a gravitational potential is unlikely to release amounts of energy sufficient to produce the observed high luminosity and high energy tail of the spectrum for most of the quasars, it has been suggested in PK83 and KE86 that the relativistic particles required to account for the observed radio, X-ray and $\gamma$-ray emission may result from shock acceleration of a part of in-falling plasma via first order Fermi acceleration which transforms directed kinetic energy into relativistic particle energy and thus produces relativistic protons with high efficiency. A steady-state situation is assumed to have developed where a standing collision-less spherically symmetric shock is proposed to form.

The question of the existence of a steady shock for spherical accretion deserve some elaborate discussion and clarification. As the absence of considerable angular 
momentum in accreting material does not supports standard Rankine-Hugoniot type shock formation, assumption of a steady and standing spherically-symmetric shock for freely falling material, may apparently contradict the concept of shock formation in general fluid flow. The situation, however, is different if the relativistic protons of sufficient energy density are present around the black hole. It is worth studying what happens when freely falling supersonic (supersonic, because, as we will see later, spherical accretion with a reasonably realistic value of inflow energy and accretion rate crosses its sonic point far away, $\gtrsim$ hundreds of Schwarzschild radius or even more, from the black hole) plasma approaches close (some factor of tens of Schwarzschild radius or even less) to the black hole. Due to various possible instabilities in supersonic plasma flow, any small disruption mechanism may slow down the flow and create a "piston" which produces a shock. Thus the initial formation of shocks may be attributed either to convection of ambient relativistic particles or to dissipation in magnetic field as suggested in the literature (see PK83, $\mathrm{KE} 86$, and references therein). Once the shock is produced, it accelerates a part of the in-falling matter to relativistic energies. Infalling thermal particles with free fall velocity are then assumed to be shock-accelerated via first order Fermi acceleration (Axfrod et al. 1977; Blandford \& Ostriker 1978; Blandford \& Eichler 1987, and references therein, Kirk \& Duffy 1999, and references therein) and relativistic protons will be produced. These shock accelerated relativistic protons were assumed to be trapped within a radius comparable to the radius of the shock surface by some mechanism (possibly by small amount of stochastic magnetic fields tied to the accreting matter, which is small enough to make the hydrodynamical approximation valid to describe the flow) because had it been the case that those protons would not be confined to the central region of the quasars, spallation of nuclei in the outer gas cloud responsible for the line emission in Quasars would result in an overabundance of boron (Baldwin et al. 1977), contrary to the observation. In addition, the matter density in this region may be assumed to be sufficiently high so that the relativistic protons are depleted mainly by nuclear interactions which, possibly could set a lower limit (though not very restrictive) on the accretion rate for a given black hole mass (PK83).

It was also suggested in PK83 that if the particles accelerated at the shock were neucleons rather than electrons, then, due to their negligible Compton losses, the Compton catastrophe could be avoided.

Because of the fact that the kinetic energy of these relativistic particles are much larger than their gravitational potential energy, and because their scattering mean free path could be larger than the black hole radius ${ }^{1}$, these relativistic protons suffer practically no Compton losses and are not instantly swallowed by the black hole,

\footnotetext{
${ }^{1}$ Furthermore, the converging magnetic field, if present, is also expected to mirror the relativistic particles and help prevent them from being absorbed by the black hole, see KE86.
}

rather they scatter several times and undergo multiple inelastic nuclear collisions and produce pions $\left(\pi^{ \pm, 0}\right)$ before being captured by the black hole (PK83, KE86). Pions generated by this process decay into relativistic electrons, positrons, neutrinos and antineutrinos and produce high energy $\gamma$-rays (see Sect. 2 of Das 1999 and references therein for various particle production and decay mechanism). As the relativistic protons are assumed to lose energy only through the above mentioned nuclear collisions and since the proton-proton collision time scale $\tau_{\text {pp }}$ would, in general, be much larger compared to the free-fall time scale, a sufficiently high density of relativistic protons could be maintained which provides radially outward pressure sufficient enough to support the sustenance of a steady and standing shock. The shock is, thus, self-supported.

In a previous work (Das 1999), we have explicitly calculated the exact location (radial distance measured from the central accretor in units of Schwarzschild radius $r_{\mathrm{g}}=\frac{2 G M_{\mathrm{BH}}}{c^{2}}$, where $M_{\mathrm{BH}}$ is the mass of the black hole, $G$ is the Universal gravitational constant and $c$ is the velocity of light in vacuum) of the above mentioned steady, standing, spherically-symmetric shock in terms of only three accretion parameters, namely, the specific energy of the flow, accretion rate (scaled in units of Eddington rate) and the adiabatic index of the flow for spherically-symmetric, transonic accretion of adiabatic fluid onto a Schwarzschild black hole. By solving the set of hydrodynamic equations describing the motion of accreting material under the influence of pseudo-Schwarzschild potential proposed by Paczyński \& Wiita (1980), we have shown there that it is possible to construct a self-consistent inflow-outflow system where a part of the accreting material may be blown as spherical wind from the spherical shock surface and the mass outflow rate $R_{\dot{m}}$ (the measure of the fraction of accreting material being "kicked off" as wind) was computed in terms of various accretion as well as shock parameters. However, calculation presented in our previous work was based on the valid assumption that spherically symmetric, transonic flow onto a Schwarzschild black hole can be well approximated using a suitable modified Newtonian potential and no general relativistic calculations were performed to study the flow profile.

In this paper, we would like to construct a selfconsistent, spherically symmetric, polytropic, transonic, non-magnetized accretion-wind system using a full general relativistic description of flow structure around a Schwarzschild black hole. In doing so, we would like to perform the following steps:

(a) We will write down the set of general relativistic hydrodynamic equations of motions describing the accretion and wind in Schwarzschild metric.

(b) Incorporating a suitable equation of state, we will solve the equations to get a general flow profile for accreting material where any dynamical (flow velocity, Mach number of the flow etc.) as well as thermodynamic (flow temperature, density, pressure etc.) quantity can be explicitly calculated at any radial distance (measured from 
the accretor in units of $r_{\mathrm{g}}$ ) in terms of only three accretion parameters, namely, the total specific energy and adiabatic index of the flow and accretion rate scaled in units of Eddington rate.

(c) We will use the formula for shock location obtained in Das (1999) with the assumption that direct use of that formula in this paper would not be quite unjustified because procedure to calculate the shock location in modified Newtonian description would roughly be unchanged also in the full general relativistic framework. This is because the shock location is obtained by equating the two alternative expressions for luminosities of the same object, hence any correction term arising from the red-shift (gravitational or cosmological) would cancel out. Using the values of dynamical (and thermodynamic) variables computed in general relativistic framework in terms of three accretion parameters mentioned in (b), we will calculate the shock standoff distance. As the condition necessary for the development and sustenance of such a shock is satisfied when at shock location, the Mach number of the inflow $M_{\mathrm{sh}}$ is considerably high (high supersonic flow at the time it encounters the shock, Ellison \& Eichler 1984), we concentrate on polytropic accretion with such a set of values of conserved specific energy $\mathcal{E}$ and accretion rate (scaled in the unit of Eddington rate) $\dot{M}_{\text {Edd }}$ which produces a high shock Mach number solution.

(d) We will take this hadronic pressure-supported shock surface to be the generating surface of mass outflow. At the shock surface, density of the post-shock material as well as the post-shock flow temperature shoots up and dynamical flow velocity falls down abruptly. In other words, highly supersonic inflow becomes subsonic and accreting matter becomes shock-compressed at this surface. Matter starts getting hotter and denser and starts piling up on the shock surface. The post-shock relativistic hadronic pressure and thermal pressure (pressure generated by high temperature produced at the shock) then gives a kick to the piled-up matter, the result of which is the ejection of outflow from the shock surface. Thus a hot and dense spherical shock surface serves as the "effective" physical atmosphere regarding the generation of mass outflow from matter accreting onto black holes. At the shock, entropy is generated and the outflow as well as post-shock inflow will have higher entropy density for the same specific energy. For this type of inflow, accretion is expected to proceed smoothly after a shock transition, since successful subsonic solutions have been constructed for accretion onto black holes embedded within normal stars with the boundary condition $u=c$ (Flammang 1982); where $u$ is the in-fall velocity of matter and $c$ is the velocity of light in vacuum.

It is to be mentioned here that one fundamental criterion for formation of hydrodynamic outflows is that the outflowing wind should have a positive Bernoulli constant which means that the matter in the post-shock region is able to escape to infinity. However, positiveness in Bernoulli's constant may lead to another situation as well where shock may quasi-periodically originate at some certain radius and propagate outwards without formation of outflows. So formation of outflows is one of the possible scenarios when we focus on the positive energy solutions. In this paper we concentrate only on solutions producing outflows, thus we use only the positive Bernoulli constant throughout our model which is standard practice for studying hydrodynamic winds. Another assumption made in this paper is to treat the accreting as well as post-shock matter as a single temperature fluid, the temperature of which is basically characterized by proton temperature.

The plan of this paper is as follows. In next section we describe how to formulate and solve the governing equations. In Sect. 3, we present our results. In Sect. 4, the results will be reviewed and conclusions will be drawn.

\section{Governing equations and solution procedure}

\subsection{Inflow model}

We assume that a Schwarzschild type black hole spherically accretes fluid obeying a polytropic equation of state. The density of the fluid is $\rho(r), r$ being the radial distance measured in the unit of Schwarzschild radius $r_{\mathrm{g}}$. We also assume that the accretion rate (in the unit of Eddington rate $\dot{M}_{\text {Edd }}$ ) is not a function of $r$ and we ignore the selfgravity of the flow. For simplicity of calculation, we choose the geometric unit where the unit of length is scaled in units of $r_{\mathrm{g}}$, units of velocity in units of $c$. All other physically relevant quantities can be expressed likewise. We also set $G=c=1$ in the system of units used here.

For a Schwarzschild metric of the form

$\mathrm{d} s^{2}=\mathrm{d} t^{2}\left(1-\frac{1}{r}\right)-\mathrm{d} r^{2}\left(1-\frac{1}{r}\right)^{-1}-r^{2}\left(\mathrm{~d} \theta^{2}+\sin ^{2} \theta \mathrm{d} \phi^{2}\right)$

the energy momentum tensor $T^{\mu \nu}$ for a perfect fluid can be written as

$T^{\mu \nu}=\epsilon u^{\mu} u^{\nu}+p\left(u^{\mu} u^{\nu}-g^{\mu \nu}\right)$

where $\epsilon$ and $p$ are proper energy density and pressure of the fluid (evaluated in the local inertial rest frame of the fluid) respectively and $u^{\mu}$ is the four velocity commonly known as

$u^{\mu}=\frac{\mathrm{d} x^{\mu}}{\mathrm{d} s}$

Equations of motion which are to be solved for our purpose are,

1) Conservation of mass flux or baryon number conservation:

$\left(\rho u_{\mu}\right)_{; \mu}=0$

and

2) Conservation of momentum or energy flux (general relativistic Euler equation obtained by taking the four divergence of $\left.T^{\mu \nu}\right)$ :

$(\epsilon+p) u_{\mu ; \nu} u^{\nu}=-p_{, \mu}-u_{\mu} p_{, \nu} u^{\nu}$

where the semicolons denote the covariant derivatives. 
Following Michel (1972), one can rewrite Eqs. (3a) and $(3 \mathrm{~b})$ for spherically symmetric flow as

$4 \pi \rho u r^{2}=\dot{M}_{\text {in }}$

and

$$
\left(\frac{p+\epsilon}{\rho}\right)^{2}\left(1-\frac{1}{r}+u^{2}\right)=C
$$

as two fundamental conservation equations for timeindependent hydrodynamical flow of matter on to a Schwarzschild black hole without back-reaction of the flow on to the metric itself. $\dot{M}_{\text {in }}$ being the mass accretion rate and $C$ is some constant (related to the total enthalpy influx) to be evaluated for a specific equation of state.

For polytropic equation of state i.e.,

$p=K \rho^{\gamma_{\text {in }}}$

(where $\gamma_{\text {in }}$ is the adiabatic index of the inflow) and defining $\dot{\mathcal{M}}$ (where $\dot{\mathcal{M}}=\dot{M}_{\text {in }} \gamma_{\text {in }} \frac{1}{\gamma_{\text {in }}-1} K^{\frac{1}{\gamma_{\text {in }}-1}}$ is a measure of the specific entropy of the flow) to be another conserved quantity of the flow except at the shock location (because shock generates entropy), one can rewrite conservation Eqs. (4a) and (4b) as (Das \& Sarkar 2001, and references therein):

$\mathcal{E}=h u_{\mathrm{t}}=\left(\frac{p+\epsilon}{\rho}\right)\left(\frac{1-\frac{1}{r}}{1-u^{2}}\right)^{\frac{1}{2}}$

and

$\dot{\mathcal{M}}=4 \pi\left[\frac{\left(\gamma_{\text {in }}-1\right) a^{2}}{\gamma_{\text {in }}-\left(a^{2}+1\right)}\right]^{\left(\frac{1}{\gamma_{\text {in }}-1}\right)} u u_{\mathrm{t}} r^{2}$

where $\mathcal{E}, h, u_{\mathrm{t}}$ and $a$ are the total conserved specific energy (which include the rest mass of matter), specific enthalpy $\left(h=\left[\frac{\gamma_{\mathrm{in}}-1}{\gamma_{\mathrm{in}}-\left(a^{2}+1\right)}\right]\right)$, specific binding energy and local adiabatic sound speed respectively. Equation (5b) may be considered as the outcome of the conservation of mass and entropy along the flow line. The expression for the adiabatic sound speed a can be written as (Weinberg 1972; Frank et al. 1992):

$a=\left(\frac{\partial p}{\partial \epsilon}\right)_{\mathcal{S}}^{\frac{1}{2}}=\sqrt{\frac{\gamma_{\mathrm{in} p}}{\rho}}=\sqrt{\frac{\gamma_{\mathrm{in}} \kappa T}{\mu \mathrm{m}_{\mathrm{H}}}}$

where $T$ is the flow temperature, $\mu$ is the mean molecular weight and $m_{\mathrm{H}} \sim m_{\mathrm{p}}$ is the mass of the hydrogen atom and $\kappa$ is Boltzmann's constant. The subscript $\mathcal{S}$ indicates that differentiation is performed at constant specific entropy.

One can now easily derive the expression for velocity gradient $\left(\frac{\mathrm{d} u}{\mathrm{~d} r}\right)$ (by differentiating Eqs. (5a) and $(5 \mathrm{~b})$ ) as

$$
\frac{\mathrm{d} u}{\mathrm{~d} r}=\frac{u\left(1-u^{2}\right)\left[a^{2}(4 r-3)-1\right]}{2 r(r-1)\left(u^{2}-a^{2}\right)} .
$$

Since the flow is assumed to be smooth everywhere, if the denominator of Eq. (7a) vanishes at any radial distance $r$, the numerator must also vanish there to maintain the continuity of the flow. One therefore arrives at the so called "sonic point (alternately, the "critical point") conditions" by simultaneously making numerator and denominator of Eq. (7a) equal to zero and the sonic point conditions can be expressed as follows

$u_{\mathrm{c}}=a_{\mathrm{c}}=\sqrt{\frac{1}{4 r_{\mathrm{c}}-3}}$.

For a specific value of $\mathcal{E}$ and $\gamma_{\text {in }}$, location of sonic point $r_{\mathrm{c}}$ can be obtained by solving the following equation algebraically

$\begin{aligned} 64 r_{\mathrm{c}}^{3}\left(\mathcal{E}^{2}-1\right) & +16 r_{\mathrm{c}}^{2}\left(2 \mathcal{E}^{2} \varphi_{\text {in }}^{2}-9\right)+4 r_{\mathrm{c}}\left(\mathcal{E}^{2} \varphi_{\text {in }}^{2}-27\right) \\ & +27=0\end{aligned}$

where $\varphi_{\text {in }}=\left(\frac{3 \gamma_{\text {in }}+2}{\gamma_{\text {in }}-1}\right)$.

To determine the behaviour of the solution near the sonic point, one needs to evaluate the value of $\left(\frac{\mathrm{d} u}{\mathrm{~d} r}\right)$ at that point (we denote it by $\left(\frac{\mathrm{d} u}{\mathrm{~d} r}\right)_{\mathrm{c}}$ ) by applying L "Hospitals" rule in Eq. (7a). It is easy to show that $\left(\frac{\mathrm{d} u}{\mathrm{~d} r}\right)_{\mathrm{c}}$ can be obtained by solving the following quadratic equations algebraically:

$$
\begin{aligned}
& \left(\frac{\mathrm{d} u}{\mathrm{~d} r}\right)_{\mathrm{c}}^{2}+\frac{\left(\gamma_{\mathrm{in}}-1\right)\left(16 r_{\mathrm{c}}^{2}-16 r_{\mathrm{c}}-8 \gamma_{\mathrm{in}} r_{\mathrm{c}}+6 \gamma_{\mathrm{in}}+3\right)}{3 r_{\mathrm{c}}\left(4 r_{\mathrm{c}}-3\right)^{\frac{3}{2}}}\left(\frac{\mathrm{d} u}{\mathrm{~d} r}\right)_{\mathrm{c}} \\
& +\frac{\left(\gamma_{\mathrm{in}}-1\right)\left(2 r_{\mathrm{c}}-1\right)\left(24 r_{\mathrm{c}}^{2}-28 r_{\mathrm{c}}-8 r_{\mathrm{c}}^{2} \gamma_{\mathrm{in}}+4 r_{\mathrm{c}} \gamma_{\mathrm{in}}+3 \gamma_{\mathrm{in}}+6\right)}{2 r_{\mathrm{c}}\left(4 r_{\mathrm{c}}-3\right)^{\frac{5}{2}}\left(r_{\mathrm{c}}-1\right)}=0
\end{aligned}
$$

It is now quite straightforward to simultaneously solve Eqs. (5a) and (5b) to get the integral curves of the flow (curves showing variation of Mach number of the flow with radial distance) for a fixed value of $\mathcal{E}$ and $\gamma_{\text {in }}$. Detail methodology for this purpose will be discussed in Sect. 2.3.

In this work we normally use the value of $\gamma_{\text {in }}$ to be $\frac{4}{3}$. Though far away from the black hole, optically thin accreting plasma may not be treated as ultra-relativistic fluid (by the term "ultra-relativistic" and "purely nonrelativistic") we mean a flow with $\gamma=\frac{4}{3}$ and $\gamma=\frac{5}{3}$ respectively, according to the terminology used in Frank et al. (1992) in general, close to the hole it always advects with enormously large radial velocity and could be wellapproximated as ultra-relativistic flow. As because our main region of interest, the shock formation zone, always lies close to the black hole (a few tens of $r_{\mathrm{g}}$ away from the hole or sometimes even less, see results and figures in Sect. 3), we believe that it is fairly justifiable to assign the value $\frac{4}{3}$ for $\gamma_{\text {in }}$ in our work. However, to rigorously model a real flow without any assumption, a variable polytropic index having proper functional dependence on radial distance (i.e., $\gamma \equiv \gamma(r)$ ) might be considered instead of using a constant $\gamma_{\text {in }}$, and equations of motion might be formulated accordingly, which we did not attempt here for the sake of simplicity. Nevertheless, we keep our option open for values of $\gamma_{\text {in }}$ other than $\frac{4}{3}$ as well and investigated the outflow for an range of values of $\gamma_{\text {in }}$ for a specific value of $\mathcal{E}$ and $\dot{M}_{\mathrm{Edd}}$ (Fig. 5a, Sect. 3.3.3). The same kind of investigations could be performed for a variety of values of $\mathcal{E}$ and $\dot{M}_{\mathrm{Edd}}$ and a set of results may be obtained with a wide range of values of $\gamma_{\text {in }}$ which tells that our calculation is not restricted to the value $\gamma_{\text {in }}=\frac{4}{3}$ only; rather the 
model is general enough to deal with all possible value of $\gamma_{\text {in }}$ for polytropic accretion.

\subsection{Shock parameters and the outflow model}

As already mentioned, a steady, collision-less shock forms due to the instabilities in the plasma flow. We assume that for our model, the effective thickness of the shock $\Delta_{\text {sh }}$ is small enough compared to the shock standoff distance $r_{\mathrm{sh}}$, and that the relativistic particles encounter a full shock compression ratio while crossing the shock. At the shock, density of matter will shoot up and inflow velocity will drop abruptly. If $\left(\rho_{-}, u_{-}\right)$and $\left(\rho_{+}, u_{+}\right)$are the pre- and post-shock densities and velocities respectively at the shock surface, then

$\frac{\rho_{+}}{\rho_{-}}=R_{\mathrm{comp}}=\frac{u_{-}}{u_{+}}$

where $R_{\text {comp }}$ is the shock compression ratio. For high shock Mach number solution, the expression for $R_{\text {comp }}$ can be well approximated as

$R_{\text {comp }}=1.44 M_{\mathrm{sh}^{\frac{3}{4}}}$

where $M_{\mathrm{sh}}$ is the shock Mach number and Eq. (5) holds for $M_{\mathrm{sh}} \gtrsim 4.0$ (Ellison \& Eichler 1985).

In terms of various accretion parameters, shock location can be computed as (Das 1999):

$r_{\mathrm{sh}}=\frac{3 \sigma_{\mathrm{pp}} \dot{M}_{\mathrm{Edd}}}{4 \pi u_{\mathrm{sh}^{2}}{ }^{2}}\left(\frac{1-2.4 M_{\mathrm{sh}}{ }^{-0.68}}{1-3.2 M_{\mathrm{sh}}{ }^{-0.62}}\right)$

where $\sigma_{\mathrm{pp}}$ is the collision cross section for relativistic protons, $u_{\mathrm{sh}}$ and $M_{\mathrm{sh}}$ are the dynamical flow velocity and the Mach number attained at the shock location, $\dot{M}_{\text {Edd }}$ is the mass accretion rate scaled in units of Eddington rate. One can understand that

$\left(u_{\mathrm{sh}}, M_{\mathrm{sh}}\right) \equiv \zeta\left(\mathcal{E}, \dot{M}_{\mathrm{Edd}}, \gamma_{\mathrm{in}}\right)$

where $\zeta$ has some complicated non-linear functional form which cannot be evaluated analytically, but the value of $u_{\mathrm{sh}}$ and $M_{\mathrm{sh}}$ can easily be obtained in terms of $\left\{\mathcal{E}, \dot{M}_{\mathrm{Edd}}, \gamma_{\mathrm{in}}\right\}$ by numerically solving Eqs. (5a,b) and (10) (with the help of Eqs. (7a-d)) simultaneously. Hence one can write

$r_{\mathrm{sh}} \equiv \xi\left(\mathcal{E}, \dot{M}_{\mathrm{Edd}}, \gamma_{\text {in }}\right)$

where $\xi$ has some functional form other than that of $\zeta$.

In ordinary stellar mass-loss computations (Tarafder 1988 , and references therein), the outflow is assumed to be isothermal till the sonic point. This assumption is probably justified, since copious photons from the stellar atmosphere deposit momenta on the slowly outgoing and expanding outflow and possibly make the flow close to isothermal. This need not be the case for outflow from black hole candidates. Our effective boundary layer, being close to the black hole, are very hot and most of the photons emitted may be swallowed by the black hole itself instead of coming out of the region and depositing momentum onto the outflow. Thus, the outflow could be cooler than the isothermal flow in our case. We choose polytropic outflow with a different polytropic index $\gamma_{\mathrm{o}}<\gamma_{\text {in }}$ due to momentum deposition. Nevertheless, it may be advisable to study the isothermal outflow to find the behaviour of the extreme case. Modelling the isothermal outflow is in progress and will be presented elsewhere.

In our calculation we assume that essentially the postshock fluid pressure and the post-shock proton temperature controls the wind formation as well as the barionic matter content of the outflow. The presence of a collision-less steady standing spherical shock discussed in this work may randomize the directed in-fall motion and at the shock surface the individual components of the total energy of the flow (which is a combination of the kinetic, thermal and gravitational energy) gets rearranged in such a manner that the thermal energy of the post-shock matter dominates (due to enormous shock generated postshock proton temperature) over the gravitational attraction of the accretor and a part of the in-falling material is driven by thermal pressure to escape to infinity as wind. In Sect. 3.3 we show that for any shock solution, whatever the initial boundary condition obeyed by the pre-shock flow, the mass-loss rate always co-relates with the postshock proton temperature which essentially supports the validity of our assumption.

The adiabatic post-shock sound speed $a_{\mathrm{sh}}^{+}$and the post-shock temperature $T_{\mathrm{sh}}^{+}$(which is basically the temperature of the protons according to our one-temperature fluid approximation) can be calculated as:

$$
a_{\mathrm{sh}}^{+}=\sqrt{\frac{\gamma_{\mathrm{o}} p_{\mathrm{sh}}^{+}}{\rho_{\mathrm{sh}}^{+}}}
$$

and

$$
T_{\mathrm{sh}}^{+}=\frac{\mu \mathrm{m}_{\mathrm{p}} \mathrm{p}_{\mathrm{sh}}^{+}}{\kappa \rho_{\mathrm{sh}}^{+}}
$$

where $p_{\mathrm{sh}}^{+}$and $\rho_{\mathrm{sh}}^{+}$are the post-shock pressure and density of the flow at shock location $r_{\mathrm{sh}}$ respectively. For low energy accretion ("cold" inflow, so to say) which is appropriate to produce a high shock Mach number solution, one can assume that the pre-shock thermal pressure $\left(p_{\mathrm{sh}}^{-}\right)$ may be neglected compared to its post-shock value $\left(p_{\mathrm{sh}}^{+}\right)$ and to the pre-shock ram pressure $\left(\rho_{\mathrm{sh}}^{-}\left(u_{\mathrm{sh}}^{-}\right)^{2}\right)$. One can obtain the value of $p_{\mathrm{sh}}^{+}$using Eqs. (8), (9) and from the total pressure balance condition at shock as,

$p_{\mathrm{sh}}^{+}=\left(u_{\mathrm{sh}}^{+}\right)^{2} r_{\mathrm{sh}}\left(\frac{R_{\mathrm{comp}}-1}{R_{\mathrm{comp}}}\right)$. 
Combining Eqs. (8)-(12), post-shock sound velocity and temperature obtained at the shock surface can be rewritten as:

$$
\begin{aligned}
a_{\mathrm{sh}}^{+}= & 0.606 \dot{M}_{\mathrm{Edd}} \sigma_{\mathrm{pp}}{ }^{1.5} \gamma_{\mathrm{o}}^{0.5}\left(u_{\mathrm{sh}}^{+}\right)^{-1.5} \\
& \times\left(1-0.694 M_{\mathrm{sh}}{ }^{-0.75}\right)^{0.5}\left(\frac{1-2.4 M_{\mathrm{sh}}{ }^{-0.68}}{1-3.2 M_{\mathrm{sh}}{ }^{-0.62}}\right)^{1.5}
\end{aligned}
$$

and,

$$
\begin{aligned}
T_{\mathrm{sh}}^{+}= & 0.014\left(\frac{\mu m_{\mathrm{p}} \dot{M}_{\mathrm{Edd}} \sigma_{\mathrm{pp}}^{3}\left(u_{\mathrm{sh}}^{+}\right)^{-3}}{\kappa}\right) \\
& \times\left(1-0.694 M_{\mathrm{sh}}{ }^{-0.75}\right)\left(\frac{1-2.4 M_{\mathrm{sh}}{ }^{-0.68}}{1-3.2 M_{\mathrm{sh}}{ }^{-0.62}}\right)^{3}
\end{aligned}
$$

The two conservation equations governing the outflow will exactly be the same as Eqs. (5a) and (5b) with different polytropic index, i.e., with $\gamma=\gamma_{\mathrm{o}}$ for outflow. So we can write,

$\mathcal{E}^{\prime}=h^{\prime} u_{\mathrm{t}}^{\prime}$

and

$\dot{\mathcal{M}}^{\prime}=4 \pi\left[\frac{\left(\gamma_{\mathrm{o}}-1\right) a_{2}^{\mathrm{o}}}{\gamma_{\mathrm{o}}-\left(a_{\mathrm{o}}^{2}+1\right)}\right]^{\left(\frac{1}{\gamma_{\mathrm{o}}-1}\right)} u_{\mathrm{o}} u_{\mathrm{t}} r^{2}$

where $\mathcal{E}^{\prime}$ is the specific energy (including rest mass) of the outflow which is also assumed to be constant throughout the flow and $\dot{\mathcal{M}}^{\prime}=\dot{M}_{\text {out }} \gamma_{\mathrm{o}} \frac{1}{\gamma_{\mathrm{o}}-1} K^{\mathrm{o}} \frac{1}{\gamma_{\mathrm{o}}-1}$ is the entropy accretion rate of the outflow. $\gamma_{\mathrm{o}}<\gamma_{\text {in }}$ as already mentioned.

Also Eq. (4a) can now be written as the mass conservation equation for the outflow as:

$\dot{M}_{\text {out }}=4 \pi \rho^{\mathrm{o}} u^{\mathrm{o}}(r)^{2}$.

In Eq. (15), $\rho^{\mathrm{o}}$ and $u^{\mathrm{o}}$ are the density and dynamical flow velocity of the outflowing material. We then define the mass outflow rate $R_{\dot{m}}$ as:

$R_{\dot{\mathrm{m}}}=\frac{\dot{M}_{\mathrm{out}}}{\dot{M}_{\mathrm{in}}}$

Like Eqs. (7a)-(7d), the value of $\frac{\mathrm{d} u^{\circ}}{\mathrm{d} r}$, sonic point conditions and the value of $\frac{\mathrm{d} u^{\circ}}{\mathrm{d} r}$ at the outflow sonic point can be written as:

$$
\begin{aligned}
& \left(\frac{\mathrm{d} u^{\mathrm{o}}}{\mathrm{d} r}\right)=\frac{u^{\mathrm{o}}\left[1-\left(u^{\mathrm{o}}\right)^{2}\right]\left[\left(a^{\mathrm{o}}\right)^{2}(4 r-3)-1\right]}{2 r(r-1)\left[\left(u^{\mathrm{o}}\right)^{2}-\left(a^{\mathrm{o}}\right)^{2}\right]} \\
& u_{\mathrm{c}}^{\mathrm{o}}=a_{\mathrm{c}}^{\mathrm{o}}=\sqrt{\frac{1}{4 r_{\mathrm{c}}^{\mathrm{o}}-3}} \\
& \left(4 r_{\mathrm{c}}^{\mathrm{o}}\right)^{3}\left[\left(\mathcal{E}^{\prime}\right)^{2}-1\right]+\left(4 r_{\mathrm{c}}^{\mathrm{o}}\right)^{2}\left[2\left(\mathcal{E}^{\prime}\right)^{2} \varphi_{\mathrm{o}}^{2}-9\right] \\
& +4 r_{\mathrm{c}}^{\mathrm{o}}\left[\left(\mathcal{E}^{\prime}\right)^{2} \varphi_{\mathrm{o}}^{2}-27\right]+27=0
\end{aligned}
$$

where $\varphi_{\mathrm{o}}=\left(\frac{3 \gamma_{\mathrm{o}}+2}{\gamma_{\mathrm{o}}-1}\right)$

$$
\begin{aligned}
& \left(\frac{\mathrm{d} u^{\mathrm{o}}}{\mathrm{d} r}\right)_{\mathrm{c}}^{2}+\frac{\left(\gamma_{\mathrm{o}}-1\right)\left[16\left(r_{\mathrm{c}}^{\mathrm{o}}\right)^{2}-16 r_{\mathrm{c}}^{\mathrm{o}}-8 \gamma_{\mathrm{o}} r_{\mathrm{c}}^{\mathrm{o}}+6 \gamma_{\mathrm{o}}+3\right]}{3 r_{\mathrm{c}}^{\mathrm{o}}\left(4 r_{\mathrm{c}}^{\mathrm{o}}-3\right)^{\frac{3}{2}}}\left(\frac{\mathrm{d} u^{\mathrm{o}}}{\mathrm{d} r}\right)_{\mathrm{c}} \\
& +\frac{\left(\gamma_{\mathrm{o}}-1\right)\left(2 r_{\mathrm{c}}^{\mathrm{o}}-1\right)\left[24\left(r_{\mathrm{c}}^{\mathrm{o}}\right)^{2}-28 r_{\mathrm{c}}^{\mathrm{o}}-8\left(r_{\mathrm{c}}^{\circ}\right)^{2} \gamma_{\mathrm{o}}+4 r_{\mathrm{c}}^{\mathrm{o}} \gamma_{\mathrm{o}}+3 \gamma_{\mathrm{o}}+6\right]}{2 r_{\mathrm{c}}^{\mathrm{o}}\left(4 r_{\mathrm{c}}^{\mathrm{o}}-3\right)^{\frac{5}{2}}\left(r_{\mathrm{c}}^{\mathrm{o}}-1\right)}=0
\end{aligned}
$$

where the superscript $o$ indicates that the quantities are measured for the outflows.

\subsection{Simultaneous solution of inflow-outflow equations}

In this work, we are interested in finding the ratio of $\dot{M}_{\text {out }}$ to $\dot{M}_{\text {in }}$ (Eq. (16)), and not the explicit value of $\dot{M}_{\text {out }}$. Also note that the primary goal of our present work was to compute the outflow rate and to investigate its dependence on various inflow parameters but not to study the collimation procedure of the outflow.

Before we proceed in detail, a general understanding of the transonic inflow outflow system in the present case is essential to understand the basic scheme of the solution procedure. Let us consider the transonic accretion first. Infalling matter becomes supersonic after crossing a saddle-type sonic point, the location of which is determined by $\left\{\mathcal{E}, \dot{M}_{\mathrm{Edd}}, \gamma_{\text {in }}\right\}$. This supersonic flow then encounters a shock (if present), location of which $\left(r_{\mathrm{sh}}\right)$ is determined from Eq. (10). At the shock surface, part of the incoming matter, having a higher entropy density (because shock in a fluid flow generates entropy), is likely to return as wind through a sonic point other than the point through which it just entered. Thus a combination of transonic topologies, one for the inflow and other for the outflow (passing through a different sonic point and following topology completely different that of the "self-wind" of the accretion), is required to obtain a full solution. So it turns out that finding a complete set of self-consistent inflow outflow solutions ultimately boils down to locate the sonic point of the polytropic outflow and the mass flux through it. Thus a supply of parameters $\mathcal{E}, \dot{M}_{\text {Edd }}, \gamma_{\text {in }}$ and $\gamma_{\mathrm{o}}$ make a self-consistent computation of $R_{\dot{m}}$ possible. Here $\gamma_{0}$ is supplied as free parameter because the selfconsistent computation of $\gamma_{\mathrm{o}}$ directly using $\mathcal{E}, \dot{M}_{\mathrm{Edd}}$ and $\gamma_{\text {in }}$ has not been attempted in this work; instead we put a constrain that $\gamma_{\mathrm{o}}<\gamma_{\text {in }}$ always and for any value of $\gamma_{\text {in }}$. In reality, $\gamma_{\mathrm{o}}$ is directly related to the heating and cooling processes taking place in the outflow.

We obtain the inflow sonic point $r_{\mathrm{c}}$ by solving Eq. (7c). Using the fourth order the Runge Kutta method, $u(r)$, $a(r)$ and the inflow Mach number $\left[\frac{u(r)}{a(r)}\right]$ are computed along the inflow from the inflow sonic point $r_{\mathrm{c}}$ till the position where the shock forms. The shock location is calculated by solving Eq. (10). Various shock parameters (i.e., density, pressure etc at the shock surface) are then computed self-consistently.

For outflow, with the known value of $\mathcal{E}^{\prime}$ and $\gamma_{\mathrm{o}}$, it is easy to compute the location of the outflow sonic point $r_{\mathrm{c}}^{\mathrm{o}}$ from Eq. (17c). At the outflow sonic point, the outflow velocity $u_{\mathrm{c}}^{\mathrm{o}}$ and polytropic sound velocity $a_{\mathrm{c}}^{\mathrm{o}}$ is computed from Eq. (17b). Using Eqs. (17a) and (17d), $\left(\frac{\mathrm{d} u^{\circ}}{\mathrm{d} r}\right)$ and 


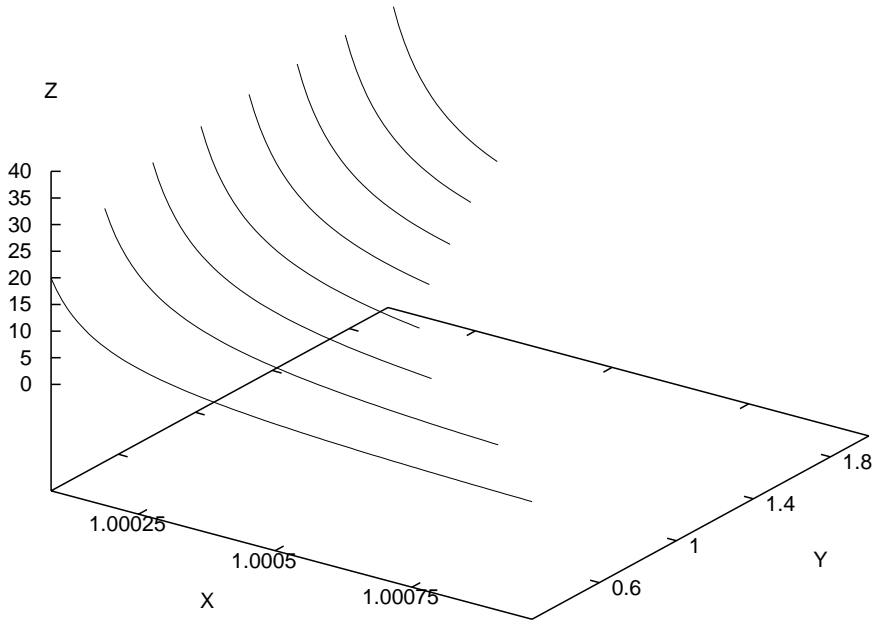

Fig. 1. Variation of shock location $r_{\mathrm{sh}}$ (in units of $r_{\mathrm{g}}$ ) with conserved specific energy $\mathcal{E}$ (which includes rest mass energy) and accretion rate $\dot{M}_{\text {Edd }}$ (scaled in units of Eddington rate) of the inflow for a fixed value of inflow adiabatic index $\gamma_{\text {in }}\left(=\frac{4}{3}\right)$. While $\mathcal{E}$ and $\dot{M}_{\text {Edd }}$ are plotted along $X$ and $Y$ axes respectively, $r_{\mathrm{sh}}$ is plotted along the $Z$ axis. It is observed that $r_{\mathrm{sh}}$ correlates with $\dot{M}_{\text {Edd }}$ and anti-correlates with $\mathcal{E}$.

$\left(\frac{\mathrm{d} u^{\mathrm{o}}}{\mathrm{d} r}\right)_{\mathrm{c}}$ is computed as was done for the inflow. RungeKutta method is then employed to integrate from the outflow sonic point $r_{\mathrm{c}}^{\mathrm{o}}$ towards the black hole to find out the outflow velocity $u^{\mathrm{o}}$ and density $\rho^{\mathrm{o}}$ at the shock location. The mass outflow rate $R_{\dot{\mathrm{M}}}$ is then computed using Eq. (16).

It is obvious from the above discussion that $R_{\dot{m}}$ should have some complicated non-linear functional dependence on the following accretion and shock parameters:

$R_{\dot{\mathrm{m}}}=\boldsymbol{\Psi}\left(\mathcal{E}, \dot{M}_{\mathrm{Edd}}, r_{\mathrm{sh}}, M_{\mathrm{sh}}, R_{\mathrm{comp}}, \gamma_{\mathrm{in}}, \gamma_{\mathrm{o}}\right)$.

As $r_{\mathrm{sh}}, M_{\mathrm{sh}}$ and $R_{\text {comp }}$ can be found in terms of $\mathcal{E}, \dot{M}_{\mathrm{Edd}}$ and $\gamma_{\text {in }}$ only, ultimately it turns out that:

$R_{\dot{\mathrm{m}}}=\boldsymbol{\Omega}\left(\mathcal{E}, \dot{M}_{\mathrm{Edd}}, \gamma_{\mathrm{in}}, \gamma_{\mathrm{o}}\right)$

where $\boldsymbol{\Omega}$ has some complicated functional form which cannot be evaluated analytically.

\section{Results}

\subsection{Shock location as a function of $\mathcal{E}$ and $\dot{M}_{E d d}$}

For a particular value of $\mathcal{E}, \dot{M}_{\mathrm{Edd}}$ and $\gamma_{\text {in }}$, the shock location (measured from the black hole in units of $r_{\mathrm{g}}$ ) can be calculated using Eq. (10). As $u_{\text {sh }}$ and $M_{\text {sh }}$ is a function of $\mathcal{E}, \dot{M}_{\mathrm{Edd}}$, and $\gamma_{\mathrm{in}}, r_{\mathrm{sh}}$ will also change with the change of any of these accretion parameters. In Fig. 1, we show the variation of $r_{\mathrm{s}}$ with $\mathcal{E}$ and $\dot{M}_{\text {Edd }}$ for a fixed $\gamma_{\text {in }}=\frac{4}{3}$. While $\mathcal{E}$ and $\dot{M}_{\text {Edd }}$ are plotted along $X$ and $Y$ axes respectively, $r_{\mathrm{sh}}$ is plotted along $Z$ axis in units of $r_{\mathrm{g}}$. It is interesting to note that different curves start and terminate at different points, which indicates that shock formation is not a generic phenomena, i.e., shock does not form for any value of $\mathcal{E}$ and $\dot{M}_{\mathrm{Edd}}$; rather, a specific region of

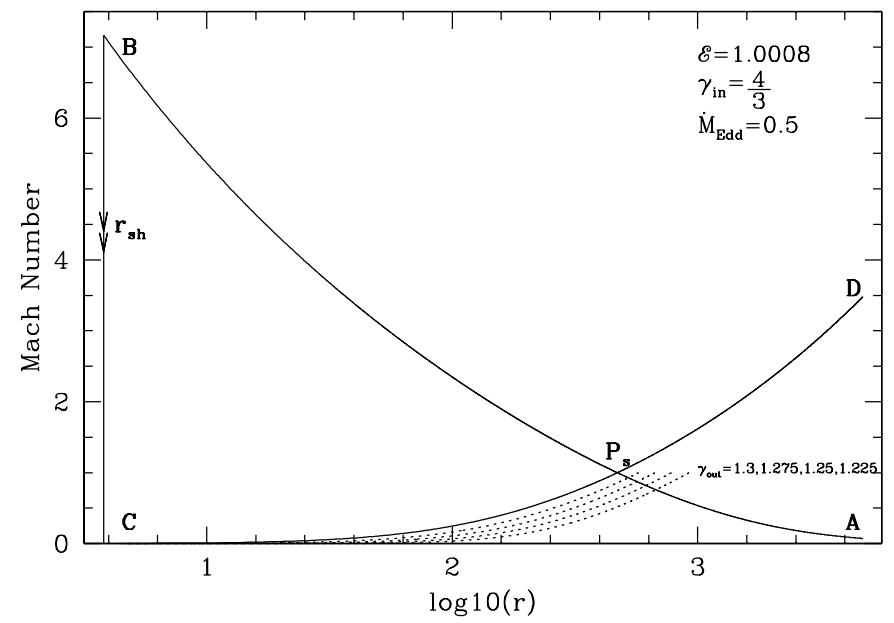

Fig. 2. Combined solution topology for transonic accretionwind system for fixed values (shown in the figure) of accretion parameters $\mathcal{E}, \dot{M}_{\mathrm{Edd}}, \gamma_{\text {in }}$ and for four outflow polytropic indices $\gamma_{\mathrm{o}}=1.3,1.275,1.25$ and 1.225 respectively (from left to right). Solid curve marked with $\mathrm{AB}$ represents the preshock transonic accretion while four dotted curves represent the accretion-powered outflow branches. Solid vertical line BC (marked by $r_{\mathrm{sh}}$ ) with double arrow stands for the shock transition and solid line marked by CD stands for the "self-wind" branch. $P_{\mathrm{s}}$ is the location of the inflow sonic point. See text for details.

parameter space spanned by $\left\{\mathcal{E}, \dot{M}_{\text {Edd }}, \gamma_{\text {in }}\right\}$ allows shock formation. Also we find that depending on the value of $\mathcal{E}$, both sub-Eddington as well as super-Eddington accretion allows shock formation for a fixed value of $\gamma_{\text {in }}$. It is evident from the figure that while shock location non-linearly anti-correlates with $\mathcal{E}$ (for a given value of $\dot{M}_{\text {Edd }}$ and $\gamma_{\text {in }}$ ), it correlates with $\dot{M}_{\text {Edd }}$ (for a fixed $\mathcal{E}$ and $\gamma_{\text {in }}$ ). Also we see that the maximum value of energy $\mathcal{E}_{\text {Max }}$ for which the shock forms decreases with an increase of $\dot{M}_{\text {Edd }}$ and also the maximum as well as minimum value of $\dot{M}_{\text {Edd }}$ for which the shock forms, decreases and increases respectively with an increase in $\mathcal{E}$ for a fixed $\gamma_{\text {in }}$. Families of curves for other values of $\gamma_{\text {in }}$ can also be obtained in the same manner.

\subsection{Combined integral curves of motion}

Figure 2 shows a typical solution which combines the accretion and the outflow. While Mach number is plotted along the $Y$ axis, the distance (in units of $r_{\mathrm{g}}$ ) from the event horizon of the accreting black hole is plotted along the $X$ axis in logarithmic scale. The accretion parameters used are $\mathcal{E}=1.0008, \dot{M}_{\mathrm{Edd}}=0.5$ and $\gamma_{\text {in }}=\frac{4}{3}$ corresponding to ultra-relativistic inflow. The solid curve AB represents the pre-shock region of the inflow and the solid vertical line $\mathrm{BC}$ with double arrow at $r_{\mathrm{sh}}$ represents the shock transition. Shock location $\left(3.8 r_{\mathrm{g}}\right)$ is obtained using the Eq. (10) for a particular set of inflow parameters mentioned above. Four dotted curves show the four different outflow branches corresponding to the four different adiabatic indices $\gamma_{0}$ of the outflow. From left to right, the 


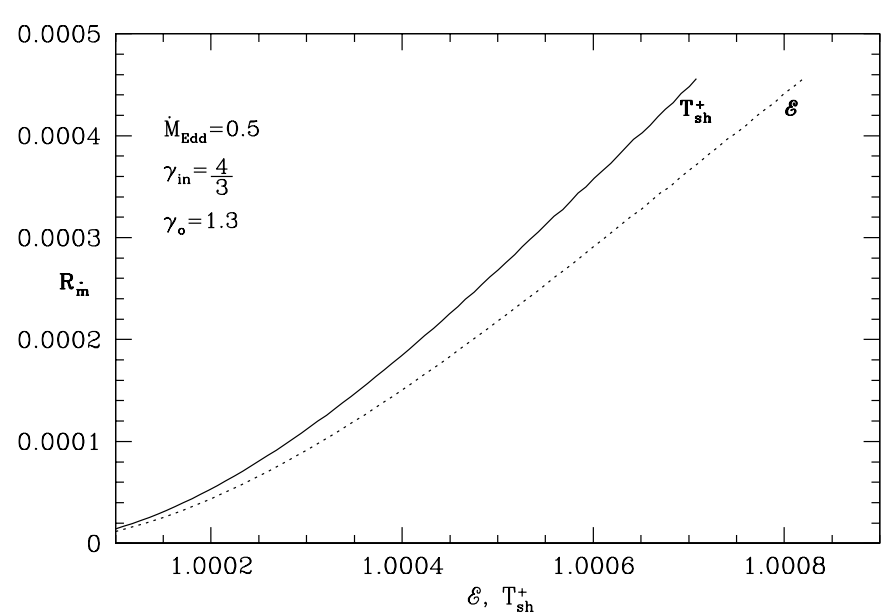

Fig. 3. Variation of $R_{\mathrm{m}}$ with conserved total specific energy (which includes the rest mass energy) $\mathcal{E}$ (dotted curve in the figure marked with $\mathcal{E}$ ) and post shock temperature (solid curve marked with $T_{\mathrm{sh}}^{+}$) of the flow for a fixed value of accretion rate and polytropic indices of the flow having values shown in the figure. Both $\mathcal{E}$ and $T_{\text {sh }}^{+}$are plotted along $X$ axis with the following scaling: $T_{\mathrm{sh}}^{+} \longrightarrow\left[1+0.0004\left(\frac{T_{\mathrm{sh}}^{+}}{T_{11}}\right)\right]$ (where $T_{11}=$ $\left.10^{11} \mathrm{~K}\right)$, to fit in the same graph.

values of $\gamma_{\mathrm{o}}$ are $1.3,1.275,1.25$ and 1.225 respectively with respective mass-outflow rates as $0.0002,0.00006,0.000011$ and 0.00000122 respectively which indicates that for a given value of $\mathcal{E}, \dot{M}_{\mathrm{Edd}}$ and $\gamma_{\mathrm{in}}, R_{\mathrm{in}}$ correlates with $\gamma_{\mathrm{o}}$, which is explicitly shown in Fig. 6. It is evident from the figure that the outflow moves along the solution curves in a completely different way to that of the "self-wind" solution of the inflow (solid line marked by CD). Also, the sonic points for all the outflowing branches are different to those of the accretion "self-wind" system which is designated as $P_{\mathrm{s}}$. While $P_{\mathrm{s}}=470.8 r_{\mathrm{g}}$, the sonic points of the outflowing branches corresponding to $\gamma_{\mathrm{o}}=1.3,1.275,1.25$ and 1.225 are 574.96, 669.7, 783.34 and $922.3 r_{\mathrm{g}}$ respectively, which indicates that the outflow sonic point increases with a decrease in the adiabatic index of the outflow and thus the wind starts with a very low bulk velocity which is why the mass-loss rate decreases. It is also observed that the sonic point of the accretion- "self-wind" system is, in general, located closer to the event horizon compared to the outflow sonic point for all values of $\mathcal{E}, \dot{M}_{\mathrm{Edd}}, \gamma_{\mathrm{in}}$ and $\gamma_{\mathrm{o}}$.

\subsection{Dependence of $R_{\dot{m}}$ on various flow parameters}

\subsubsection{Variation of $R_{\dot{m}}$ with $\mathcal{E}$}

In Fig. 3, we have plotted the variation of $R_{\dot{m}}$ with conserved inflow specific energy (which includes the rest mass energy of matter) $\mathcal{E}$ (dotted line marked with $\mathcal{E}$ ) for a fixed value of $\dot{M}_{\mathrm{Edd}}(=0.5), \gamma_{\mathrm{in}}\left(=\frac{4}{3}\right)$ and $\gamma_{\mathrm{o}}(=1.3)$. We observe that the mass-outflow rate non-linearly correlates with $\mathcal{E}$. The explanation may be as follows:

As $\mathcal{E}$ increases, $r_{\text {sh }}$ decreases (see Fig. 1, Sect. 3.1) and the post-shock bulk velocity of the flow $u_{\mathrm{sh}}^{+}$as well as the

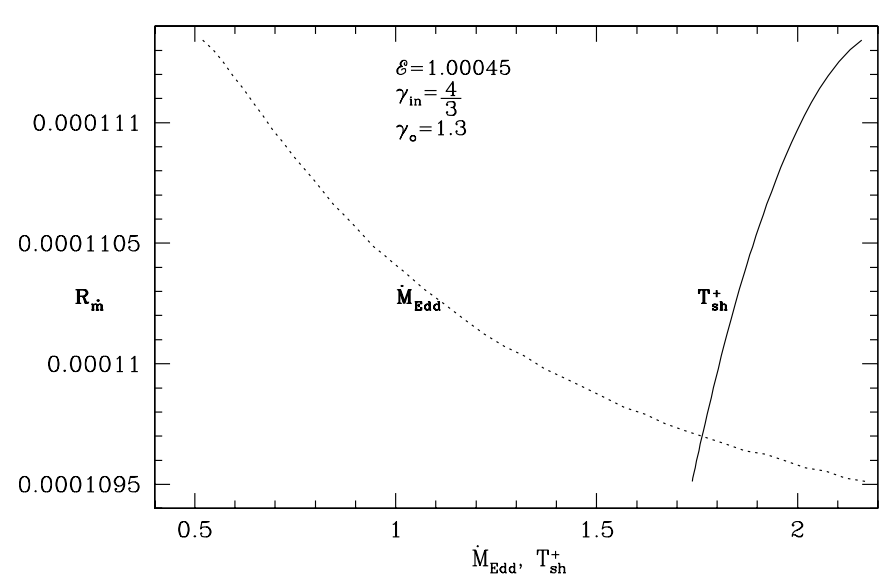

Fig. 4. Variation of $R_{\mathrm{m}}$ with accretion rate (dotted line marked $\dot{M}_{\text {Edd }}$ ) and corresponding post-shock flow temperature (solid line in the figure marked by $T_{\mathrm{sh}}^{+}$) for fixed values of $\mathcal{E}, \gamma_{\mathrm{in}}$, and $\gamma_{\mathrm{o}}$ shown in the figure. $T_{\mathrm{sh}}^{+}$is scaled as $T_{\mathrm{sh}}^{+} \longrightarrow 2.2\left(\frac{T_{\mathrm{sh}}^{+}}{T_{11}}\right)$. It is evident from the figure that low-luminosity objects produce more mass-loss, see text for details.

post-shock density $\rho_{\mathrm{sh}}^{+}$increases. The outflow rate, which is the product of three quantities $r_{\mathrm{sh}}, \rho_{\mathrm{sh}}^{+}$and $u_{\mathrm{sh}}^{+}$(see Eq. (15)), increases in general due to the combined "tug of war" of these three quantities. Moreover, the closer the shock is to the black hole, the greater the amount of gravitational potential will be available to be put onto the relativistic protons to provide stronger outward pressure and the closer the shock forms to the black hole, the higher is the post-shock temperature (the effective characteristic outflow temperature) and the higher is the amount of outflow (as the wind is observed to be strongly thermally driven, see discussion below). Thus the mass-outflow rate increases with $\mathcal{E}$ because for a particular set of fixed values of $\dot{M}_{\text {Edd }}, R_{\dot{\mathrm{m}}}$ is proportional to $\dot{M}_{\text {out }}$ which increases with $\mathcal{E}$. Also in the same figure, we show the variation of $R_{\dot{\mathrm{m}}}$ with post-shock temperature $T_{\mathrm{sh}}^{+}$(solid line in the figure marked with $T_{\mathrm{sh}}^{+}$). The value of $T_{\mathrm{sh}}^{+}$corresponds to the values of $\mathcal{E}$ shown in the same figure and is scaled as $T_{\mathrm{sh}}^{+} \longrightarrow\left[1+0.0004 \times\left(\frac{T_{\mathrm{sh}}^{+}}{T_{11}}\right)\right]\left(\right.$ where $\left.T_{11}=10^{11} \mathrm{~K}\right)$ to fit in the same graph. We see that post-shock temperature correlates with the energy of the flow, and for a fixed accretion rate and adiabatic indices of the inflow and outflow, $R_{\dot{\mathrm{m}}}$ also correlates with post-shock temperature, which indicates that outflow is thermally driven as well. If we draw a family of curves for $R_{\dot{\mathrm{m}}}$ vs. $\mathcal{E}$ (alternatively, $R_{\dot{\mathrm{m}}}$ vs. $T_{\mathrm{sh}}^{+}$) for different values of $\dot{M}_{\mathrm{Edd}}$ (figure not presented in this paper) ranging from sub-Eddington to super-Eddington accretion rate, we observe that the maximum value of $R_{\dot{m}}$ obtained for a fixed $\dot{M}_{\text {Edd }}$ (while $\mathcal{E}$ is being varied) increases with a decrease of $\dot{M}_{\text {Edd }}$ and also the maximum value of $T_{\mathrm{sh}}^{+}$follows the same trend as mentioned above. So a "high energy-low luminosity" combination for polytropic accretion maximizes the post-shock flow temperature and gives rise to the highest amount of outflow. 


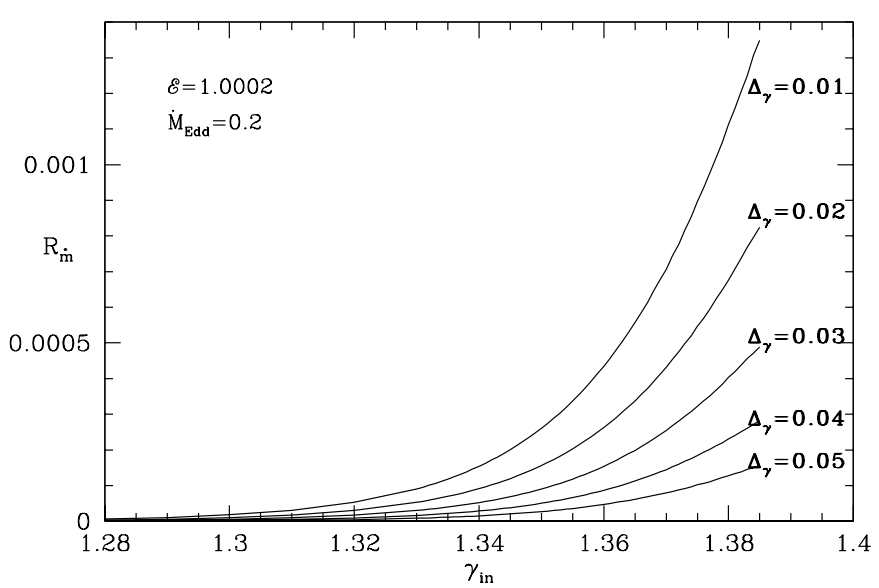

Fig. 5a. For fixed values of energy and accretion rate shown in the figure, variation of $R_{\mathrm{m}}$ with polytropic index of the inflow $\left(\gamma_{\text {in }}\right)$ is presented for five different values of $\Delta_{\gamma}=\gamma_{\text {in }}-\gamma_{\mathrm{o}}$, where $\gamma_{\mathrm{o}}$ is the polytropic index of the outflow. It is observed that ultra-relativistic flows produce less mass-loss compared to pure-nonrelativistic flows, see text for details.

\subsubsection{Variation of $R_{\dot{m}}$ with $\dot{M}_{\text {Edd }}$}

In Fig. 4 we show the variation of $R_{\mathrm{m}}$ as a function of accretion rate (dotted curve marked with $\dot{M}_{\text {Edd }}$ ) scaled in units of Eddington rate for a fixed value of $\mathcal{E}(=1.00045)$ $\gamma_{\text {in }}\left(=\frac{4}{3}\right)$ and $\gamma_{\mathrm{o}}(=1.3)$.

It is observed that $R_{\dot{m}}$ anticorrelates with $\dot{M}_{\text {Edd }}$ which indicates that low-luminosity flow produces more massloss. Here $R_{\mathrm{m}}$ correlates with post shock temperature $T_{\mathrm{sh}}^{+}$ (solid curve marked with $T_{\mathrm{sh}}^{+}$). $T_{\mathrm{sh}}^{+}$shown here is scaled as $T_{\mathrm{sh}}^{+} \longrightarrow 2.2 \times\left(\frac{T_{\mathrm{sh}}^{+}}{T_{11}}\right)$ to fit in the same graph. The values of $T_{\text {sh }}^{+}$correspond to the varying $\dot{M}_{\text {Edd }}$ shown in this graph. It is also obvious from this figure that outflow is thermally driven because $R_{\dot{m}}$ correlates with $T_{\mathrm{sh}}^{+}$. It can be shown that while for a fixed value of $\mathcal{E}$ and $\gamma_{\mathrm{in}}, r_{\mathrm{sh}}, \rho_{\mathrm{sh}}^{+}$and $u_{\mathrm{sh}}^{+}$ correlates with $\dot{M}_{\text {Edd }}$, shock Mack number, compression ratio and post-shock flow temperature anti-correlates with the accretion rate. However, from the figure we see that unlike $\mathcal{E}$, variation of $R_{\dot{\mathrm{m}}}$ is somewhat insensitive to the variation of $\dot{M}_{\text {Edd }}$ for fixed $\mathcal{E}$ and $\gamma_{\text {in }}$.

\subsubsection{Variation of $R_{\dot{m}}$ with adiabatic indices}

In previous cases, the polytropic index $\gamma_{\text {in }}$ of the accreting matter was always kept fixed at the value $\frac{4}{3}$. To have a better insight of the behaviour of the outflow, we plot $R_{\dot{m}}$ as a function of $\gamma_{\text {in }}$ (Fig. 5a) for a fixed value of $\mathcal{E}(=1.0002)$ and $\dot{M}_{\text {Edd }}(=0.2)$. The upper range of $\gamma_{\text {in }}$ shown here is the range for which shock forms for the specified value of $\mathcal{E}$ and $\dot{M}_{\text {Edd }}$. We have chosen the value of $\gamma_{\mathrm{o}}$ in such a way so that the condition $\gamma_{\mathrm{o}}<\gamma_{\text {in }}$ is always satisfied. Defining $\Delta_{\gamma}$ to be $\Delta_{\gamma}=\gamma_{\text {in }}-\gamma_{\mathrm{o}}$, we study the variation of $R_{\dot{\mathrm{m}}}$ with $\gamma_{\mathrm{in}}$ for five values of $\Delta_{\gamma}=0.01,0.02,0.03,0.04$ and 0.05 (from top to bottom respectively). We observe that $R_{\dot{m}}$ correlates with $\gamma_{\mathrm{in}}$, which is expected because the specific enthalpy of the flow increases with $\gamma_{\text {in }}$ to produce

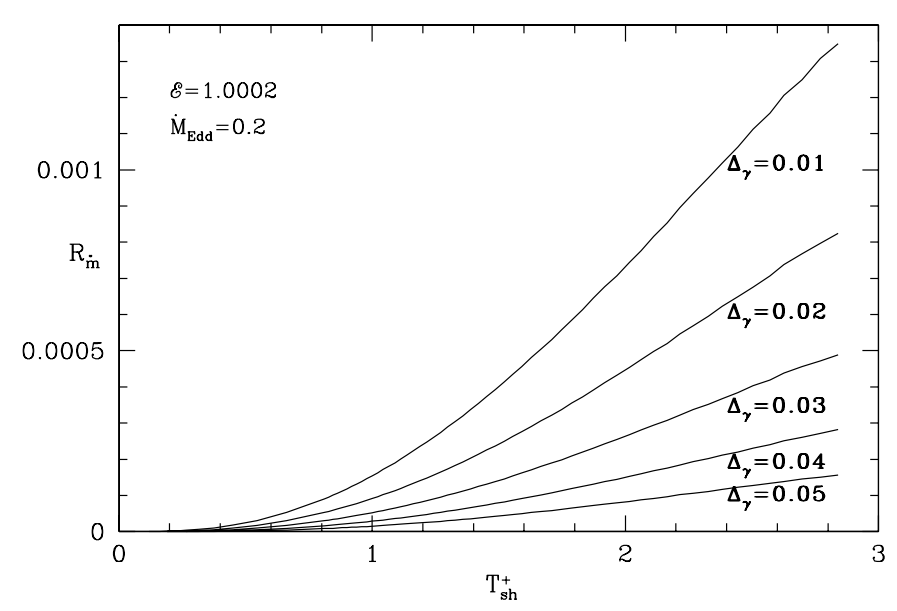

Fig. 5b. Variation of $R_{\dot{m}}$ with post-shock flow temperature $T_{\mathrm{sh}}^{+}$corresponding to values of $\gamma_{\text {in }}$ shown in Fig. 5a. Outflow is observed to be thermally driven, see text for detail.

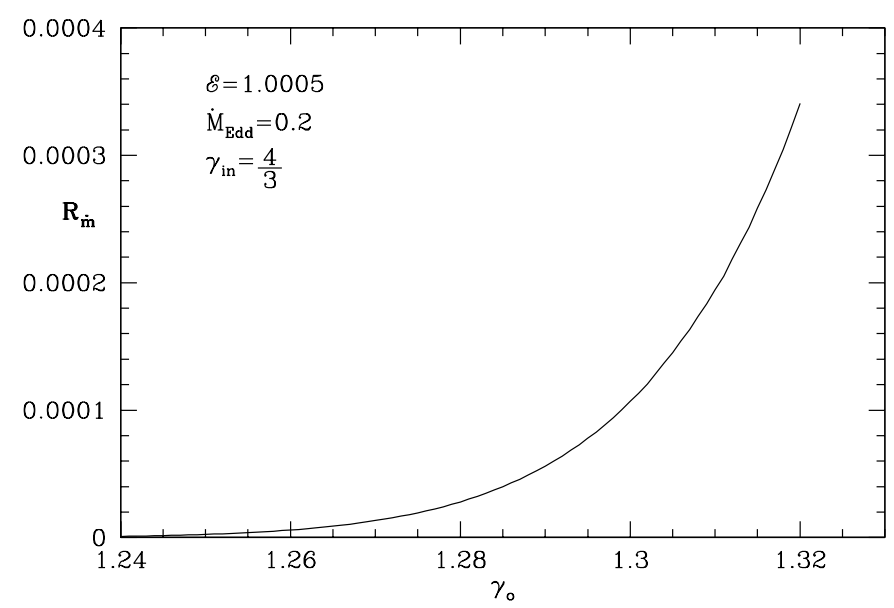

Fig. 6. Variation of $R_{\mathrm{m}}$ with polytropic index of the outflow $\gamma_{\mathrm{o}}$ for a fixed values of $\mathcal{E}, \dot{M}_{\mathrm{Edd}}$ and $\gamma_{\text {in }}$ as shown in the figure.

a higher post-shock temperature for higher value of $\gamma_{\text {in }}$ (see also Fig. 5b). We observe from our calculation that $\rho_{\text {sh }}^{+}$and $u_{\text {sh }}$ correlates while $r_{\text {sh }}$ anticorrelates with $\gamma_{\text {in }}$. So increment of $\gamma_{\text {in }}$ satisfies all possible conditions to have a high value of $R_{\mathrm{m}}$. In Fig. 5b, we show the variation of $R_{\mathrm{m}}$ with $T_{\mathrm{sh}}^{+}$(corresponding to the values of $\gamma_{\mathrm{in}}$ shown in Fig. 5a) scaled in units of $T_{11}=10^{10} \mathrm{~K}$ to show that here also the flow is thermally driven. Also, we observe that $M_{\text {sh }}$ as well as the shock compression ratio $R_{\text {comp }}$ anti correlates with $\gamma_{\text {in }}$ so that "weak-shock" solutions are preferred to obtain a high value of mass-loss for this case. So we conclude that as the accretion approaches from its ultra-relativistic nature to its non-relativistic regime, the mass-loss rate increases.

In Fig. 6, we show the variation of $R_{\dot{m}}$ with $\gamma_{\mathrm{o}}$ for a fixed value of $\mathcal{E}=1.0005, \dot{M}_{\mathrm{Edd}}=0.2$ and $\gamma_{\mathrm{in}}=\frac{4}{3}$. The general conclusion is that $R_{\dot{m}}$ correlates with $\gamma_{\mathrm{o}}$. This is because as $\gamma_{\mathrm{o}}$ increases, shock location and post-shock density of matter does not change (as $\gamma_{\mathrm{o}}$ does not have any role in shock formation or in determining the $R_{\text {comp }}$ ) but the sonic point of the outflow is pushed inward, hence 
the velocity with which outflow leaves the shock surface goes up, resulting the increment in $R_{\dot{\mathrm{m}}}$.

\section{Conclusion}

In this paper, we could successfully construct a selfconsistent spherically-symmetric, polytropic, transonic, non-magnetized inflow-outflow system by simultaneously solving the set of hydrodynamic equations governing the accretion and wind around a Schwarzschild black hole using full general relativistic framework. Introducing a steady, standing, hadronic-pressure supported spherical shock (formation of which was first proposed by KE86 and PK83) surface around the black hole as the effective physical atmosphere which may be responsible for generation of accretion-powered spherical wind, we calculate the mass-outflow rate $R_{\dot{m}}$ in terms of only three accretion parameters (conserved energy of the flow $\mathcal{E}$, which includes the rest mass energy of matter, accretion rate $\dot{M}_{\text {Edd }}$ scaled in units of Eddington rate and polytropic index of the flow $\gamma_{\text {in }}$ ) and only one outflow parameter (the polytropic index of the outflow, $\gamma_{\mathrm{o}}$ ). Not only do we provide a sufficiently plausible estimation of $R_{\dot{\mathrm{m}}}$, we could also successfully study the dependence and variation of this rate on various physical parameters governing the flow.

At this point, it is worth mentioning that the hot and dense shock surface around black holes, which is proposed here as the effective physical barrier around compact objects regarding the mass outflow, may be generated due to other physical effects as well for spherical accretion (Chang \& Ostriker 1985; Mészáros \& Ostriker 1983; Babul et al. 1989; Park 1990, 1990a). Another very important approach launched recently was to construct such an "effective barrier" for non-spherical disc accretion to introduce the concept of CENtrifugal pressure supported BOundary Layers (CENBOL). Treating the CENBOL as the effective atmosphere of the rotating flows around compact objects (which forms as a result of standing Rankine-Hugoniot shock or due to the maximization of polytropic pressure of accreting material in absence of shock), detailed computation of the mass-outflow rate from the advective accretion disks has been done, and dependence of this rate on various accretion and shock parameters has been quantitatively studied by constructing a self-consistent diskoutflow system (Das 1998; Das \& Chakrabarti 1999).

The basic conclusions of this paper are the following:

1. Shock formation is not a generic phenomena, i.e., not all solutions contain shock, rather a specific region of parameter space spanned by $\mathcal{E}, \dot{M}_{\text {Edd }}$ and $\gamma_{\text {in }}$ allows shock formation. Also we found that for given values of $\mathcal{E}, \dot{M}_{\mathrm{Edd}}$ and $\gamma_{\mathrm{in}}$, while the value of shock location (in units of $r_{\mathrm{g}}$ ) correlates with $\dot{M}_{\mathrm{Edd}}$, it anti-correlates with both $\mathcal{E}$ and $\gamma_{\text {in }}$;

2. The shock surface can serve as the "effective" physical barrier around the black hole regarding generation of mass loss via transonic spherical wind. The fraction of accreting material being blown as wind (which is denoted as $R_{\dot{m}}$ ) could be computed in terms of three accretion parameters and one outflow parameter;

3. While $R_{\dot{m}}$ correlates with $\mathcal{E}, \gamma_{\text {in }}$ and $\gamma_{\mathrm{o}}$, it anticorrelates with $\dot{M}_{\mathrm{Edd}}$, which indicates that lowluminosity objects produce more mass-loss, though outflow could be generated for both sub-Eddington as well as super-Eddington accretion;

4. If a shock forms, then whatever the initial flow conditions and whatever the nature of dependence of $R_{\dot{m}}$ on any of the accretion/ shock/ outflow parameters, $R_{\dot{m}}$ always correlates with post-shock flow temperature, which indicates that outflow is strongly thermally driven; hotter flow always produces more winds.

Our calculations in this paper, being simply founded, do not explicitly include various radiation losses and cooling processes, combined effects of which may reduce the post-shock proton temperature (which means the reduction of outflow temperature), in reality could be lower than what we have obtained here and the amount of outflow would be less than what is obtained in our calculation. This deviation will be more important for systems with high accretion rates. Nevertheless, cases of low accretion rates discussed here would not be affected that much and our preliminary investigation shows that even if we incorporate various losses, the overall profile of the various curves showing the dependence of $R_{\mathrm{m}}$ on different inflow parameters would be exactly the same, only the numerical value of $R_{\dot{m}}$ in some cases (especially for high accretion) might decrease. However, as we have shown that $R_{\dot{m}}$ anticorrelate with accretion rate (see Sect. 3.3.2, Fig. 4) and also it has been shown that the mass-loss rate does not have that strong a dependence on $\dot{M}_{\text {Edd }}$, we believe that such changes will not bring any significant change in our overall conclusions.

Our present work, as we believe, may have some important consequences regarding modelling of the astrophysical outflows emanating from galactic and extra-galactic sources powered by isolated accreting compact objects. Galactic and extra-galactic sources of jets and outflows are now widely believed to be fed by accreting black holes sitting at the dynamical centre of these sources. In the absence of any binary companion, spherically symmetric Bondi (Bondi 1952) type accretion may occur onto isolated central black hole if accreting matter has a negligible amount of intrinsic angular momentum. This may happen when the central super-massive black hole at the galactic centre is surrounded by a dense stellar cloud in such a way that the vector sum of the angular momentum of tidally disrupted matter (from a number of stars with trajectories approaching sufficiently close to the hole) almost vanishes. On the other hand, unlike the ordinary stellar bodies, black holes do not have their own "physical" atmosphere and outflows in these cases have to be generated from the accreting matter only. Hence we believe that it is necessary to study the accretion and outflow (from various astrophysical sources powered by accreting compact objects) in the same framework instead of treating the 
outflow separately from accretion phenomenon. At the same time, as the fundamental criterion for constructing any self-consistent physical model demands the minimization of the number of inputs to the model, we may conclude that modelling the astrophysical outflow needs a concrete formulation where the outflow can be described in terms of minimum number of physical parameters governing the inflow. The success of our work presented in this paper, as we believe, is precisely this. We could rigorously compute the mass outflow rate $R_{\dot{m}}$ in terms of only three inflow parameters and only one outflow parameter. To the best of our knowledge, there is no such model present in the literature which rigorously studies the accretion-powered spherical outflow in a general relativistic framework using only the four parameters mentioned above.

Although in this work we have performed our calculation for a $10 M_{\odot}$ Schwarzschild black hole, general flow characteristics will be unchanged for black hole of any mass except the fact that the region of parameter space responsible for shock formation will be shifted and the value of $R_{\dot{\mathrm{m}}}$ will explicitly depend on the mass of the black hole. In our next work, we will show the direct dependence of $R_{\mathrm{m}}$ on the mass of the black hole and will apply our model to some specific astrophysical sources of outflows to roughly estimate the amount of outflow in $M_{\odot} / \mathrm{yr}^{-1}$.

Acknowledgements. The author would like to acknowledge the hospitality provided by the Astronomy Unit, School of Mathematical Sciences, Queen Mary \& Westfield College, University of London, where a part of this research was carried out.

\section{References}

Axford, W. I. 1981, Ann. NY Acad. Sci., 375, 297

Axford, W. I., Leer, E., \& Skadron, G. 1977, 15th Int., Cosmic Ray Conf. (Plovdiv) 11, 132
Babul, A., Ostriker, J. P., \& Mészáros, P. 1989, ApJ, 347, 59

Baldwin, J., et al. 1977, A\&A, 61, 165

Begelman, M. C. 1978, A\&A, 70, 583

Blandford, R. D., \& Eichler, D. 1987, Phys. Repts., 154, 1

Blandford, R. D., \& Ostriker, J. P. 1978, ApJ, 221, L29

Blumenthal, G. R., \& Mathews, W. G. 1976, ApJ, 203, 714

Bondi, H. 1952, MNRAS, 112, 195

Brinkmann, W. 1980, A\&A, 85, 146

Chang, K. M., \& Ostriker, J. P. 1985, ApJ, 288, 428

Das, T. K. 1998, in Observational Evidence for Black Holes in the Universe, ed. S. K. Chakrabarti (Kluwer Academic: Holland), 113

Das, T. K. 1999, MNRAS, 308, 201

Das, T. K. 2001, MNRAS, submitted

Das, T. K., \& Chakrabarti, S. K. 1999, Class. Quantum Grav., 16,3879

Das, T. K., \& Sarkar, A. 2001, A\&A, 374, 1150

Ellision, D. C., \& Eichler, D. 1984, ApJ, 286, 691

Ellision, D. C., \& Eichler, D. 1985, Phys. Rev. Lett., 55, 2735

Flammang, R. A. 1982, MNRAS, 199, 833

Frank, J., King, A., \& Raine, D. 1992, Accretion Power in Astrophysics, 2nd Edition (Cambridge University Press)

Hoyle, F., \& Lyttleton, R. A. 1939, Proc. Camb. Phil. Soc., 35, 592

Kazanas, D., \& Ellison, D. C. 1986, ApJ, 304, 178 (KE86)

Kirk, J. G., \& Duffy, P. 1999, J. Phys. G, 25, R163

Mészáros, P., \& Ostriker, J. P. 1983, ApJ, 273, L59

Malec, E. 1999, Phys. Rev. D, 60, 104043

Michel, F. C. 1972, Astrophys. Space Sci., 15, 153

Paczyński, B., \& Wiita, P. J. 1980, A\&A, 88, 23

Park, M. G. 1990, ApJ, 354, 64

Park, M. G. 1990a, ApJ, 354, 83

Protheroe, R. J., \& Kazanas, D. 1983, ApJ, 256, 620 (PK83)

Tarafdar, S. P. 1988, ApJ, 331, 932

Sarkar, A., \& Das, T. K. 2001, IJMPD, in press

Shapiro, S. 1973a, ApJ, 180, 531

Shapiro, S. 1973b, ApJ, 185, 69

Weinberg, S. 1972, Gravitation and Cosmology: Principles and Applications of the General Theory of Relativity (John Wiley \& Sons) 